\title{
Bacillus cereus pneumonia in an immunocompetent patient: a case report
}

\author{
Yuichiro Shimoyama ${ }^{1 *}$, Osamu Umegaki ${ }^{1}$, Yukimasa Ooi ${ }^{2}$, Tomoyuki Aguii ${ }^{3}$, Noriko Kadono ${ }^{1}$ and Toshiaki Minami ${ }^{4}$
}

\begin{abstract}
Background: Bacillus cereus (B. cereus) rarely causes lower respiratory tract infections, although most reported cases of $B$. cereus pneumonia are fatal despite intensive antibiotic therapy. We present a case of $B$. cereus pneumonia in an immunocompetent patient.

Case presentation: An 81-year-old woman was transferred from a district general hospital to our hospital for treatment of congestive heart failure. The patient presented with a nonproductive cough, dyspnea, edema in both lower extremities, orthopnea, fever, and occult blood in the stool. A chest radiograph indicated bilateral pleural effusion and pulmonary congestion. After diuretic therapy and chest drainage, bilateral pleural effusion and pulmonary congestion improved. On day 2, she experienced severe respiratory distress. B. cereus was isolated from two blood sample cultures. On day 4, her condition had progressed to severe respiratory distress $\left(\mathrm{PaO}_{2} / \mathrm{FiO}_{2}\right.$ ratio $\left.=108\right)$. A chest radiograph and computed tomography indicated extensive bilateral infiltrates. She was transferred to the intensive care unit and was intubated. B. cereus was also isolated from five blood sample cultures at that time. After isolating B. cereus, we switched antibiotics to a combination of imipenem and levofloxacin, which were effective. She had no history of immunodeficiency, surgery, ill close contacts, risk factors for HIV or tuberculosis, recent central venous catheter insertion, or anthrax vaccination. She improved and was discharged from the intensive care unit after several days.

Conclusion: This is a rare case of $B$. cereus pneumonia in an immunocompetent patient, who subsequently recovered. Bacillus should be considered as a potential pathogen when immunocompetent patients develop severe pneumonia.
\end{abstract}

Keywords: Bacillus cereus, Pneumonia, Immunocompetent patient

\section{Background}

Bacillus cereus (B. cereus) is a Gram-positive, aerobic to facultative, spore-forming rod that is widely distributed in the environment [1]. B cereus is occasionally associated with food-borne illness, its presence in cultures is often considered a contaminant, and it is typically not further characterized beyond a descriptive identification and may not be reported at all [2]. However, severe hematogenous infections caused by $B$. cereus have been reported, especially in drug addicts, premature neonates, and patients with severe underlying diseases or compromised immunity [3]. B. cereus rarely causes lower respiratory tract infections, although most reported cases of $B$. cereus pneumonia are fatal despite intensive

\footnotetext{
* Correspondence: shimocchiliebesfreud512@yahoo.co.jp

'Department of Anesthesiology, Osaka Medical College, Intensive Care Unit, Osaka Medical College Hospital, 2-7 Daigaku-machi, Takatsuki, Osaka 569-8686, Japan

Full list of author information is available at the end of the article
}

antibiotic therapy [3]. Here we present a case of $B . c e-$ reus pneumonia in an immunocompetent patient.

\section{Case presentation}

An 81-year-old woman $(160 \mathrm{~cm}, 60 \mathrm{~kg})$ was transferred from a district general hospital to our hospital for treatment of congestive heart failure. At admission, the patient presented with a nonproductive cough, dyspnea, edema in both lower extremities, orthopnea, fever, and occult blood in the stool, all of which began to about 10 days prior to admission. Past medical history included atrial fibrillation (AF). She had a no drinking and smoking history, and activities of daily living were in the normal range. Her temperature was $37.3{ }^{\circ} \mathrm{C}$, pulse rate was 133 beats per min with $\mathrm{AF}$, respiratory rate was 24 breaths per min, and blood pressure was 115/58 $\mathrm{mmHg}$. Laboratory findings were as follows: WBC count, 11,040 cells $/ \mathrm{mm}^{3}$ without a left shift; hematocrit, 34.7\%; platelets, $145,000 / \mathrm{mm}^{3}$; creatinine, $0.8 \mathrm{mg} / \mathrm{dL}$; alanine 
aminotransferase, $69 \mathrm{U} / \mathrm{mL}$; aspartate aminotransferase, $56 \mathrm{IU} / \mathrm{mL}$; total bilirubin, $1.4 \mathrm{mg} / \mathrm{dL}$; and respiratory alkalosis. A chest radiograph indicated bilateral pleural effusion and pulmonary congestion (Fig. 1). We performed transthoracic echocardiography (TTE). TTE showed an ejection fraction of $50 \%$; verrucas were not detected. Intravenous ampicillin/sulbactam (6 g/day) therapy was initiated to treat a potential bacterial infection (Fig. 2). After diuretic therapy and chest drainage, bilateral pleural effusion and pulmonary congestion improved. On day 2, she experienced severe respiratory distress, with diminished breath sounds, bilateral pulmonary crackles, and an oxygen saturation of $98 \%$ while receiving $100 \%$ oxygen through a nonrebreather $4-\mathrm{L}$ mask. B. cereus was isolated from two blood sample cultures (arterial blood and venous blood) collected at admission (Fig. 3), and intravenous levofloxacin (250$500 \mathrm{mg}$ /day) therapy was initiated instead of ampicillin/ sulbactam therapy (Fig. 2). No pathogenic organisms were identified by sputum and pleural effusion of chest drain cultures. On day 3, clindamycin (1200 mg/day) was combined with levofloxacin (Fig. 2). On day 4, her condition progressed to severe respiratory distress $\left(\mathrm{PaO}_{2} / \mathrm{FiO}_{2}\right.$ ratio $\left.=108\right)$. A chest radiograph (Fig. 4) and computed tomography (CT) (Fig. 5) indicated extensive bilateral infiltrates. Her temperature was $37.5{ }^{\circ} \mathrm{C}$, pulse rate was 139 beats per min with $\mathrm{AF}$, blood pressure was $78 / 47 \mathrm{mmHg}$, and oxygen saturation was $93 \%$ while receiving $100 \%$ oxygen by a rebreather $15-\mathrm{L}$ mask. She was transferred to the intensive care unit (ICU) and intubated. She had pulmonary coarse crackles, and moderate amounts of frothy pale pink respiratory secretions were

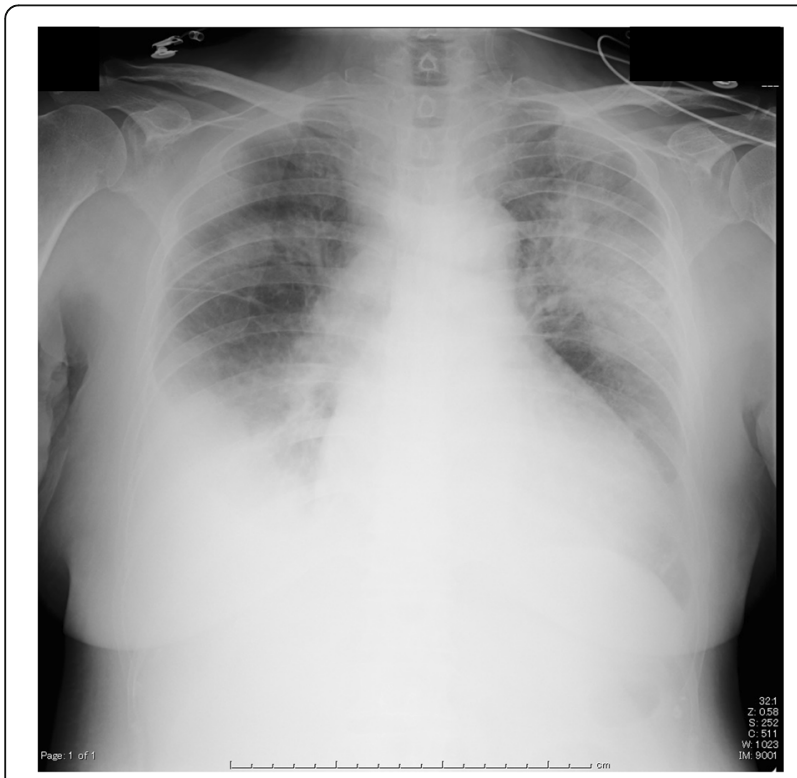

Fig. 1 Chest radiograph indicated bilateral pleural effusion and pulmonary congestion collected from the endotracheal tube. Dopamine, dobutamine, and noradrenaline were administered for hypotension. Laboratory findings were as follows: WBC count, 9860 cells $/ \mathrm{mm}^{3}$ without a left shift; hematocrit, $37.0 \%$; platelets, $147,000 / \mathrm{mm}^{3}$; creatinine, $1.47 \mathrm{mg} / \mathrm{dL}$; alanine aminotransferase, $98 \mathrm{IU} / \mathrm{mL}$; aspartate aminotransferase, $54 \mathrm{IU} / \mathrm{mL}$; total bilirubin, $1.5 \mathrm{mg} / \mathrm{dL}$; and respiratory alkalosis. The patient was unable to mount an adequate leukocyte count in light of her clinical condition. B. cereus was isolated from five blood sample cultures (four arterial blood cultures and one venous blood culture), but stool cultures were negative at that time.

On day 4, intravenous imipenem $(750 \mathrm{mg} /$ day $)$ therapy was initiated instead of levofloxacin and clindamycin therapy (Fig. 2). On day 5, levofloxacin (250-500 mg) was combined with imipenem (Fig. 2). She had no history of immunodeficiency, surgery, ill close contacts, risk factors for HIV or tuberculosis, recent central venous catheter insertion, or anthrax vaccination. On day 7 , a chest radiograph and $\mathrm{CT}$ indicated a reduction in the size of bilateral infiltrates, suggesting that respiratory therapy and antibiotic therapy were effective. The patient was extubated and, on day 9, discharged from the ICU. No further remarkable changes were noted, and she was discharged from the hospital.

\section{Discussion}

The present case was unusual, given that the patient was not immunocompromised but succumbed to lung infiltrates associated with $B$. cereus. B. cereus is a wellknown pathogen in food poisoning. It causes toxinmediated, self-limited illness characterized by emetic or diarrheal syndromes $[4,5]$. It is also known to cause bacteremia [6], endocarditis [7], meningitis [8], and pneumonia [2, 3, 9-20].

Previously reported cases of $B$. cereus pneumonia in adults are rare and usually associated with significant risk factors which are summarized in Table 1. Immunocompromised patients with $B$. cereus pneumonia frequently have septicemia and fatal illness. Most previously reported cases of infection occurred in patients with hematological disorders or alcohol abuse. However, four lethal cases of B. cereus pneumonia in immunocompetent welders and metalworkers have been reported $[2,10]$. Our patient had no history of immunodeficiency, surgery, ill close contacts, risk factors for HIV or tuberculosis, recent central venous catheter insertion, or anthrax vaccination. It is interesting that our patient, who had none of the known risk factors for severe respiratory illness, survived the episode. However, the patient's age ( 81 years) may have been a contributing factor. As age advances, the immune system undergoes profound remodeling and decline. This immune senescence predisposes older adults to a higher risk of acute 

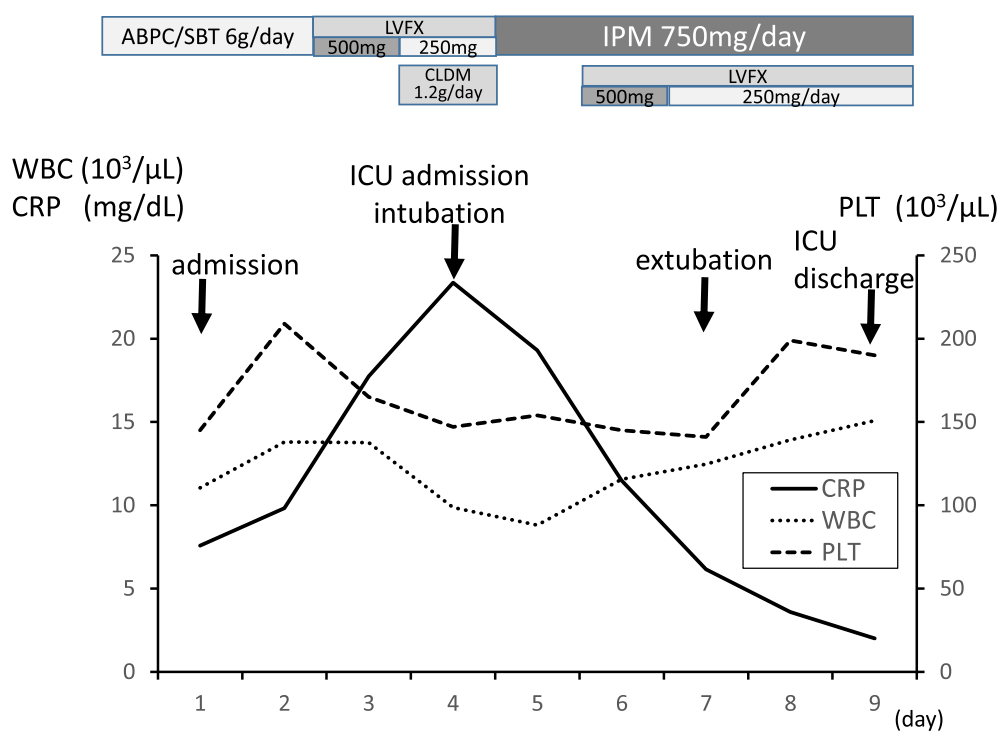

Fig. 2 Time course of laboratory data and antibiotic treatment

viral and bacterial infections [21]. Compared to previously reported episodes of $B$. cereus pneumonia in adults (Table 1), our patient was much older and thus may have been more susceptible to $B$. cereus infection.

Non-anthracis Bacillus species might be dismissed as contaminants when isolated from clinical samples. Therefore, detection of the microorganism in multiple samples is usually necessary to make a definitive diagnosis of $B$. cereus pneumonia [3].

In the present case, the pathogenic role of B. cereus in pneumonia was confirmed by seven different specimens of blood that were sampled aseptically. However, we did not perform a bronchoscopic assessment of lung infiltrates, and B. cereus was not confirmed from bronchial lavage fluid and transbronchial lung biopsy specimens.

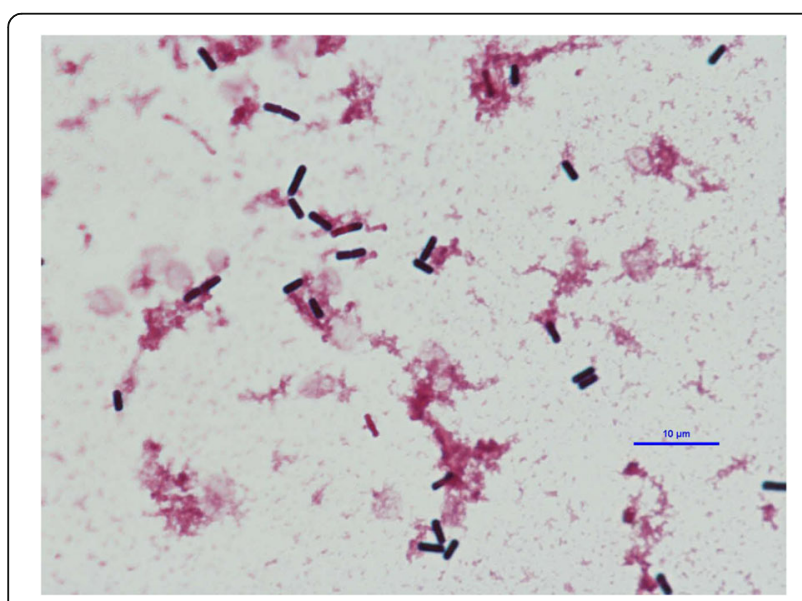

Fig. 3 Bacillus cereus was isolated from two blood sample cultures collected at admission
There were no episodes of infectious disease other than severe pneumonia during hospitalization. Respiratory symptoms such as cough and dyspnea, and gastrointestinal symptoms (occult blood in the stool) were present. Therefore, in the present case, B. cereus pulmonary infection may have resulted from transient bacteremia from a gastrointestinal infection. Indeed, we had suspected as such at a relatively early stage (on day 2 ). $B$. cereus produces $\beta$-lactamase and is therefore resistant to penicillin and cephalosporins [1]. B. cereus is usually susceptible to clindamycin, vancomycin, fluoroquinolones,

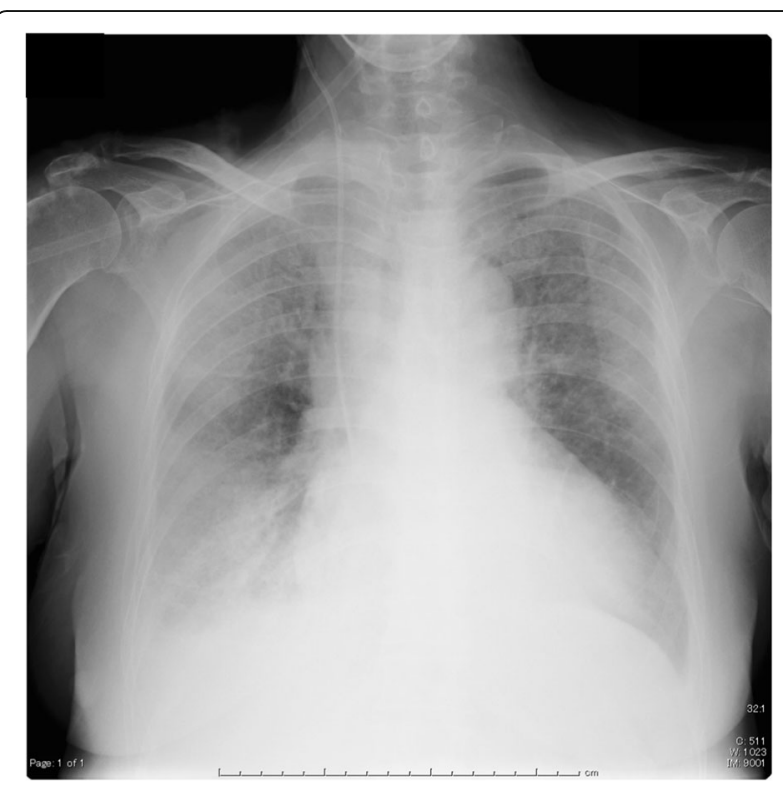

Fig. 4 Chest radiograph indicated extensive bilateral infiltrates 


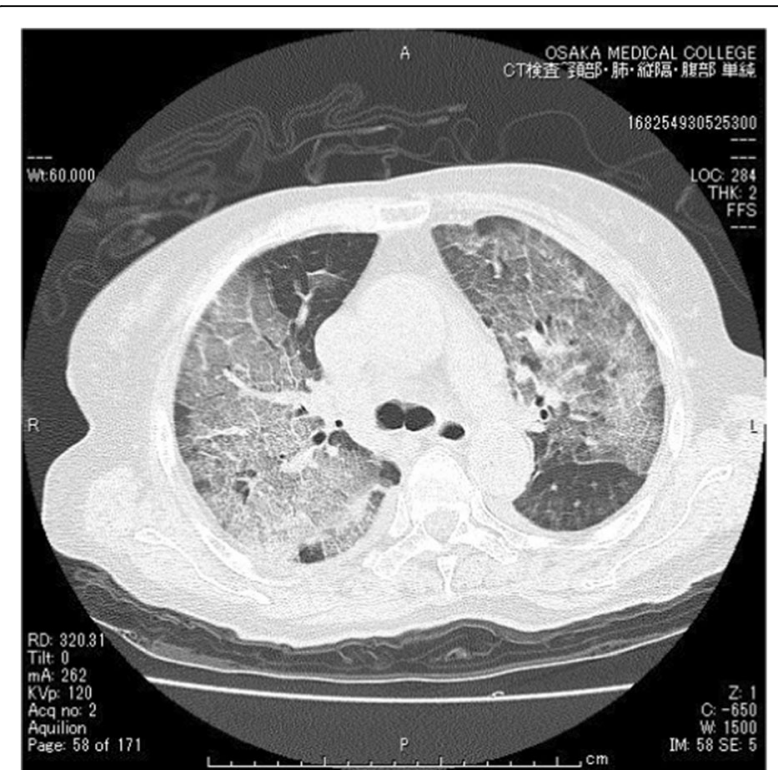

Fig. 5 Computed tomography indicated extensive bilateral infiltrates

carbapenems, and aminoglycosides. After isolating B. cereus, we switched antibiotics to a combination of imipenem and levofloxacin, which were effective.

\section{Conclusions}

In summary, we report a rare case of B. cereus pneumonia in an immunocompetent patient, who subsequently recovered. Healthcare providers should consider Bacillus species as a potential pathogen when immunocompetent patients develop severe pneumonia.

Table 1 Reported episodes of $B$. cereus pneumonia in adults

\begin{tabular}{lllll}
\hline Patient & Age & Risk factor & Outcome & Reference \\
\hline 1 & Not available & None & Died & 20 \\
2 & 52 & Leukemia & Died & 19 \\
3 & 63 & Leukemia & Died & 18 \\
4 & 29 & Leukemia & Recovered & 17 \\
5 & 60 & Alcohol abuse & Recovered & 16 \\
6 & 18 & Alcohol abuse & Recovered & 15 \\
7 & 54 & Leukemia & Recovered & 14 \\
8 & 21 & Bronchiectasis & Recovered & 13 \\
9 & 46 & None (welder) & Died & 2 \\
10 & 41 & None (welder) & Died & 2 \\
11 & 52 & Aplastic anemia & Died & 12 \\
12 & 37 & Leukemia & Died & 11 \\
13 & 39 & None (metal worker) & Died & 10 \\
14 & 56 & None (metal worker) & Died & 10 \\
15 & 60 & Leukemia & Died & 9 \\
16 & 43 & Nephrotic syndrome & Recovered & 3 \\
\hline
\end{tabular}

\section{Abbreviations}

AF: Atrial fibrillation; Bacillus cereus: B. cereus; CT: Computed tomography; ICU: Intensive care unit; TTE: Transthoracic echocardiography

\section{Acknowledgements}

None

Funding

None

Availability of data and materials

Not applicable

\section{Authors' contributions}

YS collected data and drafted the manuscript; OU, OY, TA, NK, and TM revised the manuscript. All authors read and approved the final manuscript for submission.

\section{Authors' information \\ None}

\section{Competing interests}

The authors declare that they have no competing interests.

\section{Consent for publication}

Written informed consent was obtained from the patient for the publication of this case report and accompanying images. A copy of the written consent is available for review by the Editor-in-Chief of this journal.

\section{Ethics approval and consent to participate}

Not applicable

\section{Publisher's Note}

Springer Nature remains neutral with regard to jurisdictional claims in published maps and institutional affiliations.

\section{Author details}

${ }^{1}$ Department of Anesthesiology, Osaka Medical College, Intensive Care Unit, Osaka Medical College Hospital, 2-7 Daigaku-machi, Takatsuki, Osaka

569-8686, Japan. ${ }^{2}$ Department of Internal Medicine, Osaka Medical College, Takatsuki, Japan. ${ }^{3}$ Department of Surgery, Osaka Medical College, Intensive Care Unit, Osaka Medical College Hospital, Takatsuki, Japan. ${ }^{4}$ Department of Anesthesiology, Osaka Medical College, Takatsuki, Japan.

Received: 31 January 2017 Accepted: 2 May 2017

Published online: 08 May 2017

\section{References}

1. Bottone EJ. Bacillus cereus, a volatile human pathogen. Clin Microbiol. 2010; 23:382-98.

2. Miller JM, Hair JG, Hebert M, Hebert L, Roberts Jr FJ, Weyant RS. Fulminating bacteremia and pneumonia due to Bacillus cereus. J Clin Microbiol. 1997;35: 504-7.

3. Miyata J, Tasaka S, Miyazaki M, Yoshida S, Naoki K, Sayama KA, et al. Bacillus cereus necrotizing pneumonia in a patient with nephrotic syndrome. Intern Med. 2013;52:101-4.

4. Thompson NE, Ketterhagen MJ, Bergdoll MS, Schantz EJ. Isolation and some properties of an enterotoxin produced by Bacillus cereus. Infect Immun. 1984;43:887-94.

5. Turnbull PC, Kramer JM, Jørgensen K, Gilbert RJ, Melling J. Properties and production characteristics of vomiting, diarrheal, and necrotizing toxins of Bacillus cereus. Am J Clin Nutr. 1979;32:219-28.

6. Cotton DJ, Gill VJ, Marshall DJ, Gress J, Thaler M, Pizzo PA. Clinical features and therapeutic interventions in 17 cases of Bacillus bacteremia in an immunosuppressed patient population. J Clin Microbiol. 1987;25:672-4.

7. Steen MK, Bruno-Murtha LA, Chaux G, Lazar H, Bernard S, Sulis C. Bacillus cereus endocarditis: report of a case and review. Clin Infect Dis. 1992;14: 945-6.

8. Barrie D, Wilson JA, Hoffman PN, Kramer JM. Bacillus cereus meningitis in two neurosurgical patients: an investigation into the source of the organism. J Infect. 1992;25:291-7. 
9. Katsuya H, Takata T, Ishikawa T, Sasaki H, Ishitsuka K, Takamatsu Y, et al. A patient with acute myeloid leukemia who developed fatal pneumonia caused by carbapenem-resistant Bacillus cereus. J Infect Chemother. 2009; 15:39-41.

10. Avashia SB, Riggins WS, Lindley C, Hoffmaster A, Drumgoole R, Nekomoto T, et al. Fatal pneumonia among metalworkers due to inhalation exposure to Bacillus cereus containing Bacillus anthracis toxin genes. Clin Infect Dis. 2007:44:414-6.

11. Frankard J, Li R, Taccone F, Struelens MJ, Jacobs F, Kentos A. Bacillus cereus pneumonia in a patient with acute lymphoblastic leukemia. Eur J Clin Microbiol Infect Dis. 2004;23:725-8.

12. Strauss R, Mueller A, Wehler M, Neureiter D, Fischer E, Gramatzki M, et al. Pseudomembranous tracheobronchitis due to Bacillus cereus. Clin Infect Dis. 2001;33:E39-41.

13. Gascoigne AD, Richards J, Gould K, Gibson GJ. Successful treatment of Bacillus cereus infection with ciprofloxacin. Thorax. 1991;46:220-1.

14. Sliman R, Rehm S, Shlaes DM. Serious infections caused by Bacillus species. Medicine (Baltimore). 1987;66:218-23.

15. Bekemeyer WB, Zimmerman GA. Life-threatening complications associated with Bacillus cereus pneumonia. Am Rev Respir Dis. 1985;131:466-9.

16. Panwalker AP, Trager GM. Necrotizing pneumonia and empyema caused by Bacillus cereus and Clostridium bifermentans. Am Rev Respir Dis. 1983:128: 333-4.

17. Leff A, Jacobs R, Gooding V, Hauch J, Conte J, Stulbarg M. Bacillus cereus pneumonia. Survival in a patient with cavitary disease treated with gentamicin. Am Rev Respir Dis. 1977:115:151-4.

18. Inde DC, Armstrong D. Clinical spectrum of infection due to Bacillus species. Am J Med. 1973;55:839-45.

19. Coonrod JD, Leadley PJ, Eickhoff TC. Bacillus cereus pneumonia and bacteremia. A case report. Am Rev Respir Dis. 1971;103:711-4.

20. Stopler T, Camuescu V, Voiculescu M. Bronchopneumonia with lethal evolution determined by a microorganism of the genus bacillus (B. cereus). Rum Med Rev. 1965:19:7-9.

21. Simon AK, Hollander GA, McMichael A. Evolution of the immune system in humans from infancy to old age. Proc Biol Sci. 2015;282:20143085.

\section{Submit your manuscript to a SpringerOpen ${ }^{\circ}$ journal and benefit from:}

- Convenient online submission

- Rigorous peer review

- Immediate publication on acceptance

- Open access: articles freely available online

- High visibility within the field

- Retaining the copyright to your article 